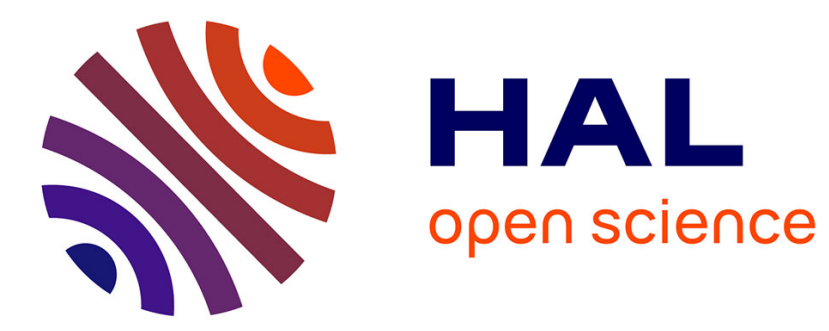

\title{
Potential of Multipass Very High Resolution SAR Interferometry for Dam Monitoring
}

Gabriel Vasile, Dider Boldo, Rémy Boudon, Guy d'Urso, Robert Muja

\section{To cite this version:}

Gabriel Vasile, Dider Boldo, Rémy Boudon, Guy d'Urso, Robert Muja. Potential of Multipass Very High Resolution SAR Interferometry for Dam Monitoring. 9th Communications International Conference, Jun 2012, Bucharest, Romania. pp.371-374, 10.1109/ICComm.2012.6262557 . hal-00785992

\section{HAL Id: hal-00785992 \\ https://hal.science/hal-00785992}

Submitted on 7 Feb 2013

HAL is a multi-disciplinary open access archive for the deposit and dissemination of scientific research documents, whether they are published or not. The documents may come from teaching and research institutions in France or abroad, or from public or private research centers.
L'archive ouverte pluridisciplinaire $\mathbf{H A L}$, est destinée au dépôt et à la diffusion de documents scientifiques de niveau recherche, publiés ou non, émanant des établissements d'enseignement et de recherche français ou étrangers, des laboratoires publics ou privés. 


\title{
Potential of Multipass Very High Resolution SAR Interferometry for Dam Monitoring
}

\author{
G. Vasile(Corresponding author) ${ }^{*}$, D. Boldo**, R. Boudon**, G. d'Urso**, R. Muja*** \\ ${ }^{*}$ GIPSA-lab / CNRS, Grenoble, France \\ ${ }^{* *} E D F, R \& D / D T G$, Chatou, France \\ ${ }^{* * *}$ ATM, Bucharest, Romania \\ *Corresponding author (E-mail : gabriel.vasile@gipsa-lab.grenoble-inp.fr)
}

\begin{abstract}
This paper presents a novel strategy for dam monitoring by repeat-pass SAR interferometry. The proposed approach couples sub-band / sub-aperture decomposition prior to the GLRT-LQ detector. This method is tested with spaceborne InSAR images provided by the TerraSAR-X satellite.

Key words - SAR; interferometry; TerraSAR-X.
\end{abstract}

\section{INTRODUCTION}

The multiplicative model has been employed for SAR data processing as a product between the square root of a scalar positive quantity (texture) and the description of an equivalent homogeneous surface (speckle). For an $m$-dimensional repeatpass SAR interferometry (InSAR) system, the single channel model has been extended as follows. In each azimuth / range location, let $\mathbf{k}$ be the $m \times 1$ complex target vector corresponding to the same area on the ground. Recent studies [1] show that the higher scene heterogeneity induced by the high-resolution spaceborne SAR systems (TerraSAR-X, TanDEM-X, COSMO-SkyMed, SAR-Lupe...) leads to nonGaussian interferometric clutter modeling.

In the case of conventional InSAR system two channels are involved and $m=2$. Denoting by $c=\rho \exp (j \phi)$ the complex correlation coefficient, the target relative displacement $d_{12}$ between the two acquisitions can be retrieved from the exact knowledge of SAR antenna phase center positions, terrain height, acquisition geometry, and an estimate of the differential interferometric phase $\phi_{12}, \rho_{12}$ is called interferometric coherence and it describes the phase stability within the estimation neighborhood. The phase information $\phi_{12}$ allows phase differences (interferograms) to be computed in order to measure topography or target displacements between repeated pass acquisitions.

In the general case, the $m$-dimensional interferometric target vector will contain information about the $m \times m$ relative displacements between each combination of 2 passes. The main parameter to estimate is the speckle covariance matrix, from which normalized correlation coefficients can be derived. Recently, a novel parameter estimation technique has been proposed in this framework [2], [3].

\section{Stable Scatterers Detection}

This section focuses on the analysis of a subset of scatterers within the scene, the so-called Stable Scatterers (SS), characterized by a deterministic point-like scattering behavior. The advantage of SS is that it is widely unaffected by multiple scattering effects and geometrical distortions allowing, as far as possible, a direct interpretation in terms of its phase center displacement. A similar approach has been proposed by the so-called Permanent Scatterers (PS) in SAR interferometry [4], where the identification of PS is based on its temporal stability (coherence over time) and relies on the availability of large time series of SAR image acquisitions. The SS differs from the PS in the sense that there is no need of temporal stability involved in its detection. The usual way to detect SS is to use Time-Frequency Distributions (Short Time Fourier Transform, Wavelet, etc) to form different sub-apertures (sublooks) or sub-bands of the same scene and to exploit their mutual correlations or coherence.

\section{A. Sub-band/sub-apertures decomposition}

When a target is illuminated by a broad-band signal and/or for a large angular extent, it is realistic to consider that the amplitude spatial repartition $I(\vec{r})$ of the scatterers depends on frequency $f$ and on aspect angle $\theta$. This repartition, denoted $I(\vec{r}, \vec{k})$, is depending on the wave vector $\vec{k}$ and it represents the energy distribution of the backscattering coefficient $H(\vec{k})$ in the hyperplane $(\vec{r}, \vec{k})$. Rewriting $I(\vec{r}, \vec{k})$ as $I(x, y, f, \theta)$, one can show that for each frequency $f_{0}$ and each angle of radar illumination $\theta_{0}, I\left(x, y, f_{0}, \theta_{0}\right)$ represents a spatial distribution of the backscattered energy for this frequency. It characterizes an "extended image" relative to the spatial repartition $I(\vec{r})$. Such images can be built using the Short Time Fourier transform (STFT) and are called hyper-images. Since the STFT is an atomic decomposition, the phase of hyper-image is preserved and it can be used for interferometric processing. Moreover, this technique decomposes the SLC signal into 2-D sub-spectra that can be interpreted as frequency sub-bands and angular sub-sectors (sub-apertures).

Consequently, the SS target with one SAR image can be reformulated in terms of hyper-image concept as a particular target (e.g. corner reflector) exhibiting a "stable" phase signal within all sub-bands / sub-apertures: given the $m$-dimensional complex target vector formed by $m$ coherent sub-bands / subapertures, the SS can be described as the product between the reference signal $\mathbf{p}=[1 \ldots 1]^{T}$ (target steering vector) times an unknown scalar complex parameter $\alpha$ (target complex amplitude). 


\section{B. Binary hypothesis test}

In this paper we propose the application of the estimation scheme presented in [2] to Stable Scatterers detection in high resolution SAR images. The SS target detection problem in compound-Gaussian clutter can be formulated as a binary hypothesis test shown in (1). Under the null hypothesis $H_{0}$, the observed target vector $\mathbf{k}$ is only the clutter $\mathbf{c}$. Under the alternative hypothesis $H_{1}$, the backscattered signal can be decomposed as the sum of the target complex signal with the clutter c. Here, the clutter is modeled as a Spherically Invariant Random Vector (SIRV).

$$
\left\{\begin{array}{l}
H_{0}: \mathbf{k}=\mathbf{c} \\
H_{1}: \mathbf{k}=\alpha \mathbf{p}+\mathbf{c}
\end{array}\right.
$$

The optimal detector under the SIRV hypothesis is given by the following relation:

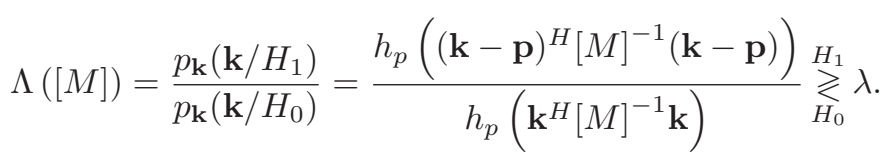
where $h_{p}(\cdot)$ is the density generator function.

\section{GLRT-LQ detector}

The Generalized Likelihood Ratio Test - Linear Quadratic (GLRT-LQ) detector can be used to detect a particular target. Let $\mathbf{p}$ be a steering vector and $\mathbf{k}$ the observed signal. The GLRT-LQ between $\mathbf{p}$ and $\mathbf{k}$ is given by [5]:

$$
\Lambda([M])=\frac{\left|\mathbf{p}^{H}[M]^{-1} \mathbf{k}\right|^{2}}{\left(\mathbf{p}^{H}[M]^{-1} \mathbf{p}\right)\left(\mathbf{k}^{H}[M]^{-1} \mathbf{k}\right)} \underset{H_{0}}{\stackrel{H_{1}}{\gtrless}} \lambda,
$$

where $[M]$ is the covariance matrix of the population under the null hypothesis $H_{0}$, i.e. the observed signal is only the clutter.

In general, the covariance matrix is unknown. One solution consists in estimating the covariance matrix $[M]$ by $[\hat{M}]_{F P}$, the fixed point covariance matrix estimator [6]. Replacing $[M]$ by $[\hat{M}]_{F P}$ in (2) leads to an adaptive version of the GLRTLQ detector.The adaptive GLRT-LQ assumes knowledge of the clutter covariance matrix and does not require any "a priori" information about the texture PDF. This detector is also reported to present the Constant False Alarm Rate (CFAR) property with respect to the texture statistical characterization, meaning that the GLRT-LQ probability of false alarm is the same for any texture statistics.

\section{Stable ScatTerers Tracking}

The relative displacement between two stable scatterers from one SLC image to another can be obtained by means of differential interferometry. The positions in the SLC images of the two scatterers are determined by employing the procedure described in the previous section. For each pixel in the SAR interferogram obtained from the two images, the phase difference $\Delta \phi_{i, j}$ can be written as: $\Delta \phi=\phi_{\text {orbital }}+\phi_{\text {topo }}+$ $\phi_{\text {disp }}+\phi_{\text {atm }}+\phi_{\text {noise }}$, where each term is a partial contribution to the total temporal phase difference.

In order to compute the displacement in Line Of Sight (LOS) of point $Q$ (the mobile SS target) with respect to the reference point $P$ (the fixed target) an additional spatial phase difference is done (between the temporal phase differences of the pixels containing the two targets). After this double phase difference, by using stable scatterers, and assuming same atmospheric conditions for neighboring points, the terms regarding the noise and the atmospheric effects are cancelled. The remaining phase difference $\Delta(\Delta \phi)$ contains two terms: one regarding the orbital and topographic differences and one provided by the displacement in LOS. The first term is given by the satellite's orthogonal baseline $B_{n}$ and the distance between the two targets (the "ground" baseline $r_{P Q}$ ). The relative displacement in LOS can be computed as in [7].

\section{A. Tracking simulation}

In order to test the tracking procedure for practical situations, a set of simulations was realized using a deformation model provided by the EDF company regarding the Puylaurent dam [8]. Considering two stable scatterers placed on the ridge of the dam, the deformation model gives the unit vector $\vec{v}$ of the direction of relative movement. If this displacement is not orthogonal to the LOS direction (characterized by the unit vector $\overrightarrow{u_{L O S}}$ ), the actual displacement can be easily computed as:

$$
D=\frac{D_{L O S}}{\overrightarrow{u_{L O S}}} .
$$

In the simulations, different displacements were randomly generated (in keeping with real data) and the phase differences were computed for various positions of the satellite relative to the master position. The initial position vectors of the two targets were considered erroneous, which leads to a false first displacement. However, one displacement is relative to the previous one, so from the second image onwards the computed displacement is correct (the systematic error is cancelled). The simulation results are summarized in Fig. 1. By not taking into account the noise and atmospheric effects in the simulations, the very small absolute error is due to the approximations made.
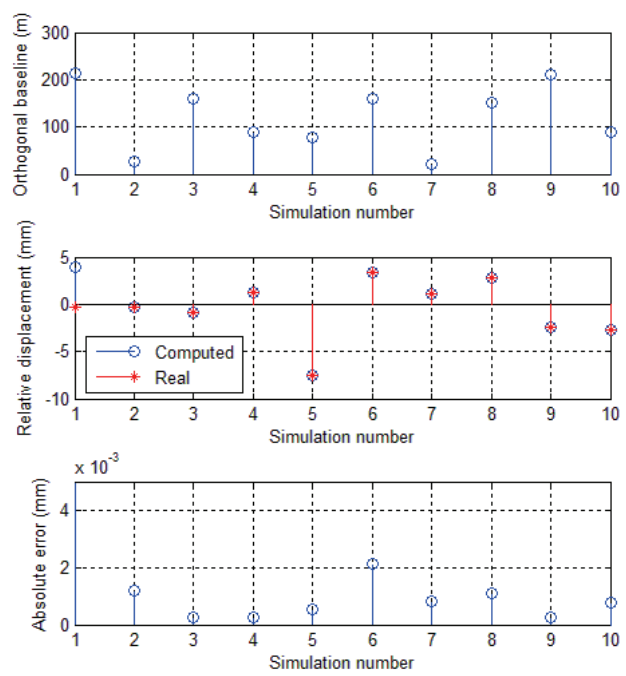

Figure 1. Puylaurent dam, France: tracking simulation results. 


\section{Results And Discussion}

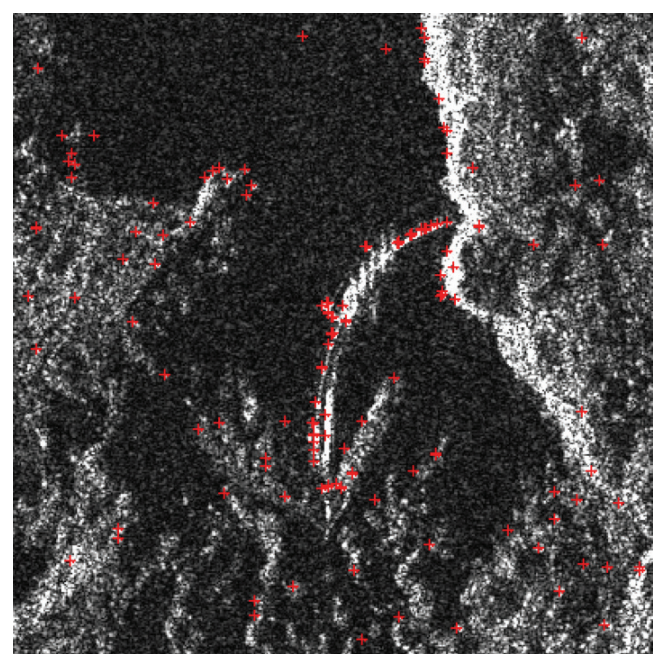

(a)

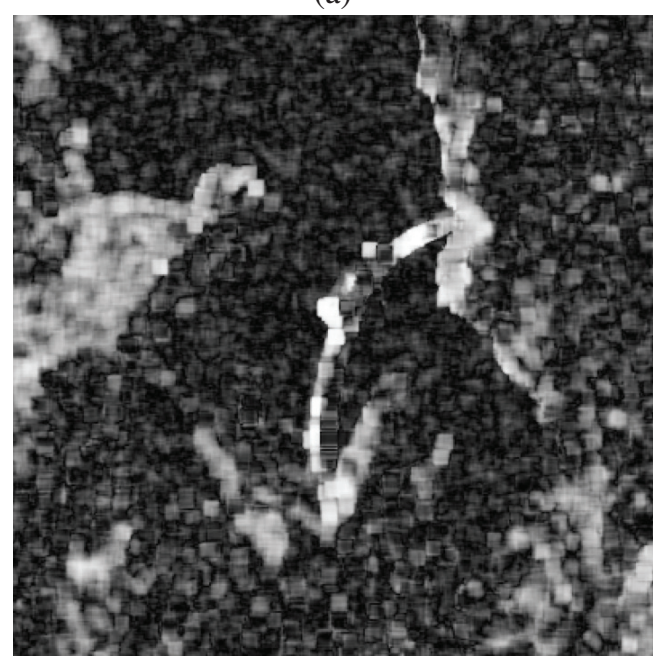

(b)

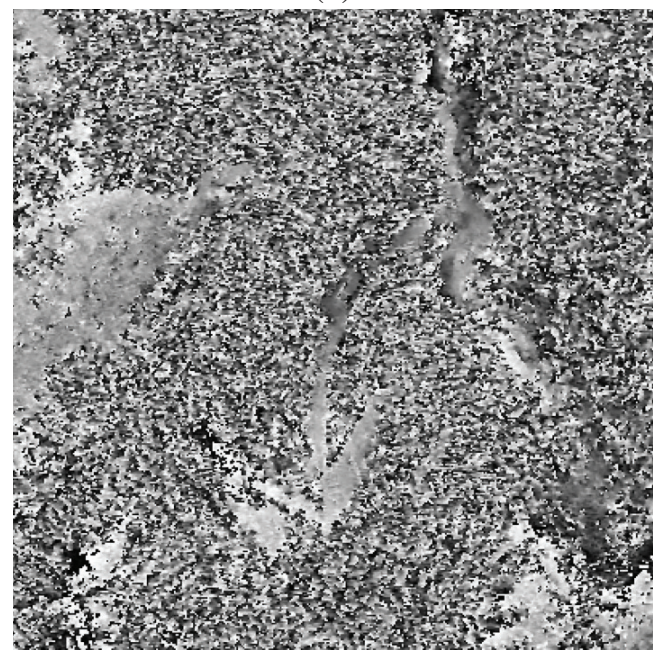

(c)

Figure 2. The Puylaurent dam, France, TerraSAR-X data, 2011. (a) detected SS image superposed over the target amplitude, texture estimated using $\widehat{\tau}$, (b) coherence map and (c) phase image.

In this section, a real data-set acquired by the TerraSAR-X satellite at X-band is analyzed: 4 stripmap (single polarization) images have been acquired over the "Chamonix Mont-Blanc" test site on 05, 16, 28 August and 21 October 2009. The best ground projected pixel spacing is respectively $2 \mathrm{~m}$ in azimuth and $2.2 \mathrm{~m}$ in range. Over the test site, two artificial targets (corner reflectors) have been installed in a geographical stable area (Refuge d'Argentière). Their initial location have been measured by differential GPS. One of the two targets has been manually moved before each acquisition using a finetunable support in the millimeter range and exact ground truth is available on the position and the displacement of the target. Fig. 3 illustrates the Line Of Sight (LOS) InSAR displacement error with respect to the "in situ" measurements. In all cases, the fixed corner reflector was used to remove the atmospheric phase delay. The obtained overall accuracy is less than 0.75 $\mathrm{mm}$ displacement in LOS.
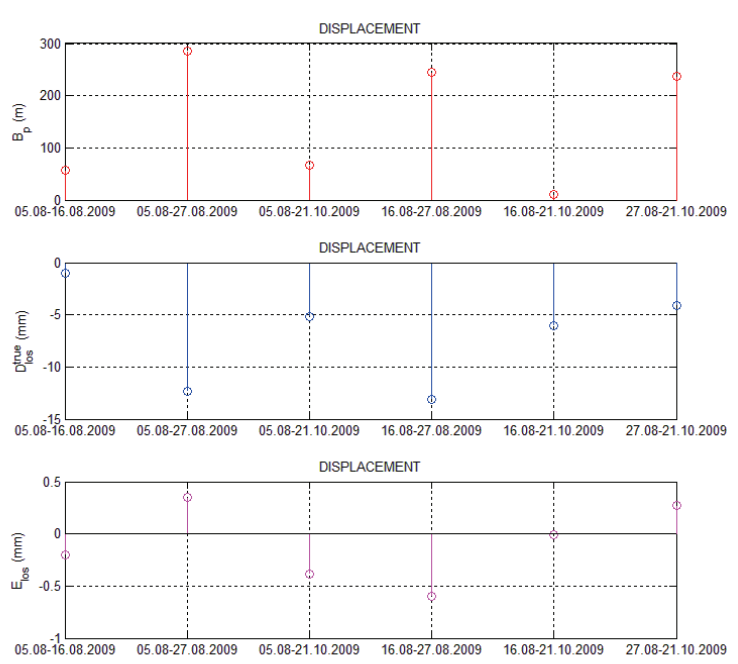

Figure 3. Refuge d'Argentière, TerraSAR-X data, 2009. (a) perpendicular interferometric baselines, (b) LOS "in situ" measured displacement, and (c) LOS interferometric displacement error.

A real data-set acquired by the TanDEM-X satellite at Xband is analyzed: 2 spotlight (ascending, single polarization, $150 \mathrm{MHz}$ bandwidth, $49^{\circ}$ incidence) images have been acquired over the "Puylaurent dam" test site on the 20th and on the 31st of July 2011. The best ground projected pixel spacing is respectively $1.1 \mathrm{~m}$ in azimuth and $1.5 \mathrm{~m}$ in range. The master data corresponds to the first acquisition that was realized in 20.07.2011 and the second data corresponds to the second acquisition that was realized after 11 days, in 31.07.2011.

Fig. 2 shows the parameter estimation results: (a) amplitude, (b) coherence map, (c) interferogram. The detection result obtained is illustrated by the red red points on Fig. 2-(a).

Finally, Fig. 4 illustrates the projected planar displacement in Lambert II cartographic coordinates. For this, the reference point has been manually selected on the dam border.

\section{CONCLUSIONS}

A novel strategy for Stable Scatterers detection was introduced by coupling sub-band / sub-aperture decomposition prior to the GLRT-LQ detector. The tracking of slowly moving 
SS targets was preformed by repeat-pass SAR interferometry. A case study with TerraSAR-X data has been presented also. This technique will be applied for the Puylaurent dam monitoring.

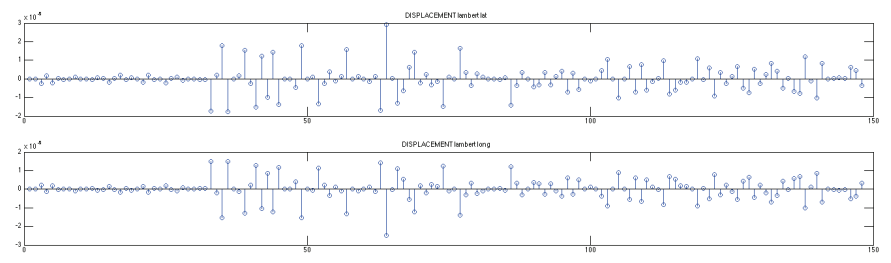

Figure 4. The Puylaurent dam, France, TerraSAR-X data, 2011: measured latitude (top) and longitude (bottom) displacement in Lambert II cartographic projection.

\section{ACKNOWLEDGMENT}

The authors would like to thank the German Aerospace Center (DLR) for providing the high-resolution TerraSAR-X stripmap SAR images through the MTH0232 and MTH0828 projects. This work was supported by the Électricité de France (EDF) company.

\section{REFERENCES}

[1] M. S. Greco and F. Gini, "Statistical analysis of high-resolution SAR ground clutter data," IEEE Transactions on Geoscience and Remote Sensing, vol. 45, no. 3, pp. 566-575, 2007.

[2] G. Vasile, J.-P. Ovarlez, F. Pascal, and C. Tison, "Coherency matrix estimation of heterogeneous clutter in high resolution polarimetric SAR images," IEEE Transactions on Geoscience and Remote Sensing, vol. 48, no. 4, pp. 1809-1826, 2010

[3] G. Vasile, F. Pascal, J.-P. Ovarlez, P. Formont, and M. Gay, “Optimal parameter estimation in heterogeneous clutter for high resolution polarimetric SAR data," IEEE Geoscience and Remote Sensing Letters, vol. 8 , no. 6, pp. 1046-1050, 2011.

[4] A. Ferretti, C. Prati, and F. Rocca, "Permanent scaterrers in SAR interferometry," IEEE Transactions on Geoscience and Remote Sensing, vol. 39, no. 1, pp. 8-20, 2001.

[5] E. Conte, M. Lops, and G. Ricci, "Asymptotically optimum radar detection in compound-Gaussian clutter," IEEE Transactions on Aerospace and Electronic Systems, vol. 31, no. 2, pp. 617-625, 1995.

[6] G. Vasile, J.-P. Ovarlez, F. Pascal, M. Gay, G. d'Urso, and D. Boldo, "Stable scatterers detection and tracking in heterogeneous clutter by repeatpass SAR interferometry," in Proceedings of the Asilomar Conference on Signals, Systems, and Computers, Pacific Grove, California, USA, 2010 pp. 1343-1347.

[7] X. Ding, Z. W. Li, J. J. Zhu, G. Feng, and J.P. Long, "Atmospheric effects on InSAR measurements and their mitigation," Sensors, vol. 8 pp. 5426-5448, 2008.

[8] G. Geffray, "Rapport GEH Loire Ardeche : barrage de Puylaurent, auscultation juin 2006 - mai 2008," Tech. Rep. 6, EDF DTG, Grenoble, France, 2008 . 